\title{
SenseWorld: Towards Cyber-Physical Social Networks
}

\author{
Raghu K. Ganti Yu-En Tsai Tarek F. Abdelzaher \\ Department of Computer Science \\ University of Illinois, Urbana-Champaign \\ Urbana, IL, USA \\ rganti2,ytsai20,zaher@cs.uiuc.edu
}

\section{Introduction}

Web-based social networks such as LinkedIn, FaceBook and MySpace have gained wide popularity in recent years. With the advent of ubiquitous sensing, future social networks will be cyber-physical, combining measured elements of the physical world with manual human input. Microsoft SensorMap [5] is an early example of a cyberphysical network in that it allows users to browse the physical world. In contrast to social networks, however, the main abstractions exported by SensorMap are those of physical objects (such as sensors), not people. This work extends the concept of SensorMap to incorporate social entities such as individuals and special-interest communities to which they belong. In the extended network, called SenseWorld, SensorMap offers a geographical index into a cyber-physical social network with its own logical topology derived from social connections.

The primary goal of SenseWorld is to facilitate connecting sensors, people and software objects to build community-centric sensing applications $[3,1]$ in a future world-wide sensor web. Such applications will rely on collection of sensory data by individuals and sharing (a redacted version of) these data towards some common goal.

To support community-centric sensing applications, the main logical abstraction of SenseWorld is a person. Other social entities, such as a local community or a special interest group, can be composed as collections of persons. Each person and each social entity in SenseWorld has a home or "headquarters" at a fixed geographic location, allowing it to be indexed by geographical browsers such as SensorMap. The person may of course be mobile. Information on their current location is always available at home.

Beneath the user interface, SenseWorld features a distributed architecture and protocol stack for sensing and information sharing. At the lowest level of that architecture are sensors affixed to static or mobile entities owned or used by a person. These sensors might, for example, be attached to cars, cell-phones, homes, or offices. They measure im- portant local context. Personal data mules, typically attached to individuals, collect context information from the surrounding sensors. Each data mule can thus archive their owner's context over time. This archived context is uploaded by the mule to the person's secure SenseWorld account, where it can be used to derive and share high-level information subject to privacy settings maintained by a privacy filter. The SenseWorld architecture defines the interfaces between the sensors, data mules, secure account services, and privacy filters.

This demonstration will showcase the human-centric sensor network abstractions, interfaces, and architectural components provided by SenseWorld, and illustrate example applications made possible by the new cyber-physical social network. These applications are supported by two main technical capabilities of SenseWorld: (i) the ability to derive high-level information from low-level data, and (ii) the ability to share information in a privacy-preserving fashion. Below, we describe the main challenges in implementing such capabilities.

\section{Technical Challenges and Solutions}

The first and foremost challenge that we face is to infer higher level information from the low level raw sensory data. As is, the raw sensory data provides little or no information regarding an individual. In order to obtain meaningful information, it is important to draw high level inferences. A general algorithm that can draw high level inferences is required. The approach we take in order to solve this problem is to use data mining techniques. We use a general frequent itemset mining algorithm to determine high level inferences from the raw sensory data collected.

The second challenge that we face is sharing the privately owned sensory data with a community of users. As one would expect, the sensory data collected can be quite sensitive. For example, GPS sensor data can potentially reveal the individual's home or work location. Thus, users may not be willing to share their sensitive sensory data. 
To reconcile the individual privacy needs with community information-sharing needs, we turn to a branch of statistics called privacy-preserving statistics. Several statistical techniques, such as those presented in [2,4], compute aggregate statistics with high accuracy given appropriately perturbed individual sensory data. In our demo, we adopt the data perturbation based approach, presented in [2], to compute aggregate community statistics.

SenseWorld adopts a client-server model where clients are the individual SenseWorld objects that store private sensory data (in a storage layer) obtained from sensors (in the sensor layer). Further, they can share true or perturbed versions of these data with external parties (subject to policies specified in the privacy layer). Servers are community objects that aggregate data over communities of interest to generate useful information made available to the clients. This architecture is pictorially depicted in Figure 1.

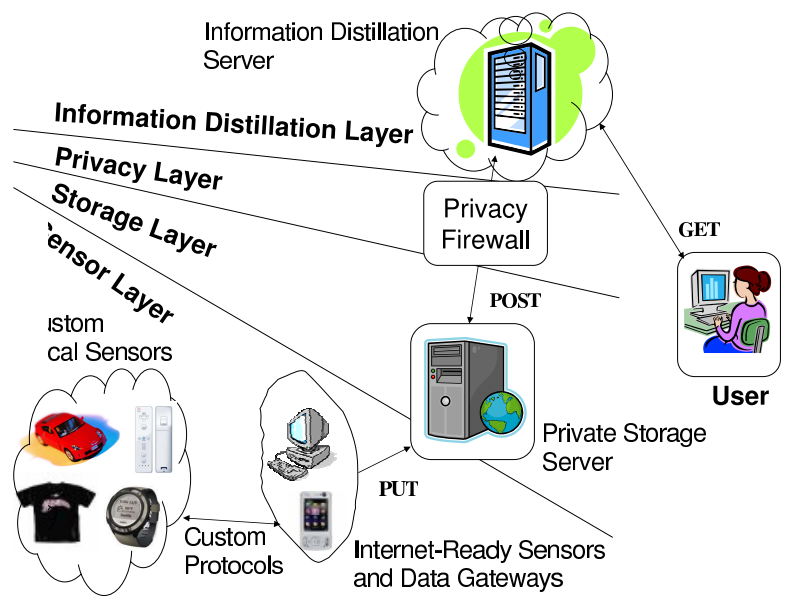

Figure 1. SenseWorld Architecture

Observe that the servers, in this architecture, act as virtual "community sensors" that can be exported via SensorMap. These community sensors can report a variety of interesting application-specific measurements from different participatory sensing applications. Similarly, clients are persons that can export virtual sensors into aspects of their private measurements. For example, an individual might export a "FindMe" sensor at their home on SensorMap that returns the latest reported physical location of the individual (except when blacked-out for privacy). The software services of SenseWorld allow easy creation of personal and community pages with well-defined data sharing policies.

\section{System Setup}

In our demo, we will be using an application that we have developed at the University of Illinois. This application collects vehicular GPS readings from sensors in the members' vehicles. The system setup consists of a vehicle that is instrumented with a GPS sensor (a MicaZ mote with an MTS420 GPS board) that samples GPS data when available and broadcasts it periodically within the vehicle.

The user wears a mote as a data mule that collects the periodic GPS broadcasts and uploads them in bulk when it comes in contact with a data gateway. In the future, we plan on replacing the latter mote with a cell phone that has a wireless data interface. The gateway uploads the data securely to a private storage server (owned by the user). The above system setup is depicted in Figure 2.

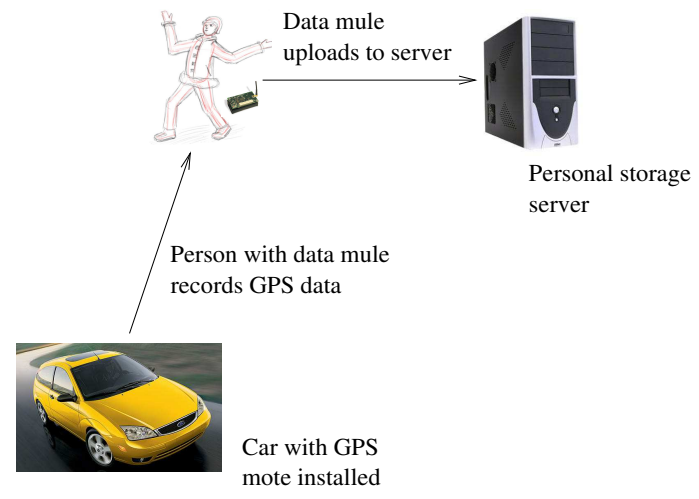

Figure 2. System Setup

In this demonstration, we will show how higher level inferences such as work patterns and frequently visited hangouts can be obtained for an individual from the raw GPS data using the above mentioned frequent itemset data mining algorithm. Further, we will also show how community information such as traffic statistics and "hot" hangouts can be obtained in a privacy-preserving manner using virtual community sensors in the above architecture.

\section{References}

[1] T. Abdelzaher, Y. Anokwa, P. Boda, J. Burke, D. Estrin, L. Guibas, A. Kansal, S. Madden, and J. Reich. Mobiscopes for human spaces. IEEE Pervasive Computing, 6(2):20-29, 2007.

[2] R. Agrawal and R. Srikant. Privacy preserving data mining. In Proceedings of the ACM SIGMOD Conference on Management of Data, pages 439-450, Dallas, TX, May 2000.

[3] S. B. Eisenman, E. Miiluzzo, N. D. Lane, R. A. Peterson, G.-S. Ahn, and A. T. Campbell. The bikenet mobile sensing system for cyclist experience mapping. In Proceedings of SENSYS, Sydney, Australia, November 2007.

[4] A. Evfimievski. Randomization in privacy preserving data mining. ACM SIGKDD Explorations Newsletter, 4(2):43-48, December 2002.

[5] Microsoft SensorMap. http://atom.research.microsoft.com/sensormap/. 